\title{
Status Report on the Instanton Counting
}

Sergey SHADCHIN

INFN, Sezione di Padova \& Dipartimento di Fisica "G. Galilei", Università degli Studi di Padova, via F. Marzolo 8, Padova, 35131, Italy

E-mail: serezha@pd.infn.it

Received December 07, 2005, in final form January 18, 2006; Published online January 22, 2006

Original article is available at http://www.emis.de/journals/SIGMA/2006/Paper008/

\begin{abstract}
The non-perturbative behavior of the $\mathcal{N}=2$ supersymmetric Yang-Mills theories is both highly non-trivial and tractable. In the last three years the valuable progress was achieved in the instanton counting, the direct evaluation of the low-energy effective Wilsonian action of the theory. The localization technique together with the Lorentz deformation of the action provides an elegant way to reduce functional integrals, representing the effective action, to some finite dimensional contour integrals. These integrals, in their turn, can be converted into some difference equations which define the Seiberg-Witten curves, the main ingredient of another approach to the non-perturbative computations in the $\mathcal{N}=2$ super Yang-Mills theories. Almost all models with classical gauge groups, allowed by the asymptotic freedom condition can be treated in such a way. In my talk I explain the localization approach to the problem, its relation to the Seiberg-Witten approach and finally I give a review of some interesting results.
\end{abstract}

Key words: instanton counting; Seiberg-Witten theory

2000 Mathematics Subject Classification: 81T60; 81T13

\section{Introduction}

The goal of this presentation is to describe some aspects of the new approach to the nonperturbative computations in the supersymmetric extension of the Yang-Mills theory $(\mathcal{N}=2$ super Yang-Mills, for the sake of brevity).

This model (with or without supplementary matter multiplets) possess a number of very interesting properties. First of all let us note that non supersymmetric Yang-Mills theory with the gauge group $\mathrm{U}(1) \times \mathrm{SU}(2) \times \mathrm{SU}(3)$ is the basis of the Standard Model, the model which describes real collider physics at the energies $\leq 100 \mathrm{GeV}$ (it is summer of 2005). Even though the perturbative computations fit well the experiment data, the non-perturbative information can still hardly be extracted from the theory. However, to describe correctly such effects as confinement we should be able to go beyond the perturbative expansion.

The problem given "as is" is too hard to be solved by existing methods. Instead, we can consider toy models which are simpler to be solvable, and at the same time enable us to gain some intuition about the state of affair. One way to get such a toy model is to consider the supersymmetric extension of the Yang-Mills theory. If we require the CPT-invariance in 4 dimensions the following supersymmetric models are possible:

- $\underline{\mathcal{N}=4}$ : It is the supersymmetric extension of the Yang-Mills theory with maximal (renormalizable) supersymmetry. It has 8 supercharges. The perturbative effects come from the 1-loop diagrams, the non-perturbative effects are trivial. It is conformal theory, that means that the $\beta$-function is zero and the running coupling constant does not run. 


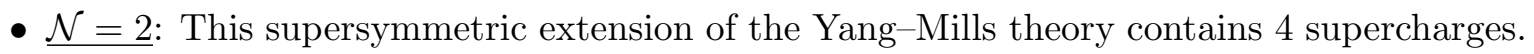
The perturbative effects are still 1-loop, but there are non-perturbative effects due to contribution of the instanton vacua. The non-perturbative effects such as confinement and the monopole condensation are present. At the same time the model contains the topological sector which allows us to compute explicitly all the non-perturbative contributions and therefore solve the model.

- $\underline{\mathcal{N}=1}$ : It is the simplest supersymmetric extension of the Yang-Mills theory. It might happen that it is the realistic model. Experiments beyond $100 \mathrm{GeV}$ will display if the Standard Model is just the supersymmetry broken version of the Minimally Supersymmetric Standard Model. This model is hard to solve, but some progress is achieved by the Dijgraaf-Vafa approach.

- $\underline{\mathcal{N}=0}$ : At present the only realistic model, but not solved yet.

From this list we see that the $\mathcal{N}=2$ super Yang-Mills theory is the only theory which is placed at the overlap of our possibility and our ambitions.

Another motivation to study this theory is its connection to the String Theory. More precisely to a theory which is believed to get rise of all superstringy models, so-called M-theory. At present the string theory computations are too hard to be performed by brute-force. However, some predictions of the M-theory are concerned to the $\mathcal{N}=2$ super Yang-Mills. Therefore if we have a method to compute the same quantities within $\mathcal{N}=2$ super Yang-Mills itself, we can check indirectly the M-theory arguments and techniques.

This report was presented on the Sixth International Conference "Symmetry in Nonlinear Mathematical Physics" (June 20-26, 2005, Kyiv).

\section{Effective action and Seiberg-Witten solution}

In this section we describe the model, its low-energy effective action and the Seiberg-Witten solution for this model.

\subsection{Microscopic description}

The microscopic action is given by the following expression (we do not include matter multiplets for the sake of brevity):

$$
\begin{aligned}
S_{\text {micro }}(X)= & \frac{\Theta}{32 \pi^{2} h^{\vee}} \int \mathrm{d}^{4} x \operatorname{Tr} F_{\mu \nu} \star F^{\mu \nu} \\
& +\frac{1}{g^{2} h^{\vee}} \int \mathrm{d}^{4} x \operatorname{Tr}\left\{-\frac{1}{4} F_{\mu \nu} F^{\mu \nu}+\nabla_{\mu} H^{\dagger} \nabla^{\mu} H-\frac{1}{2}\left[H, H^{\dagger}\right]^{2}\right. \\
& \left.+i \psi^{A} \sigma^{\mu} \nabla_{\mu} \bar{\psi}_{A}-\frac{i}{\sqrt{2}} \psi_{A}\left[H^{\dagger}, \psi^{A}\right]+\frac{i}{\sqrt{2}} \bar{\psi}^{A}\left[H, \bar{\psi}_{A}\right]\right\},
\end{aligned}
$$

where $X=\left(A_{\mu}, H, \psi_{A}\right)$ is the set of fields, $A_{\mu}(x)$ being the gauge field, $H(x)$ is the Higgs and $\psi_{A}(x)$ are two gluinos, $A$ is the extended supersymmetry index, $A=1,2$. All fields are supposed to be matrices in the adjoint representation of the Lie algebra of the gauge group. The trace $\operatorname{Tr}\{\cdot\}$ is taken over the adjoint representation and $h^{\vee}$ is the dual Coxeter number of the gauge group. $\Theta$ is the instanton angle, the first term is responsible for the strong $\mathrm{CP}$ violation in the Standard Model. Note that the first term is the topological invariant of the principle bundle whose connection is $A_{\mu}$, it does not affect to the equations of motion and its contribution to the action is $\Theta k$, where $k \in \mathbb{Z}$ is the instanton number or, mathematically speaking, the second Chern class. 
The action (1) can be written in a more compact way using the $\mathcal{N}=2$ extended superfield formalism. Let us introduce the $\mathcal{N}=2$ superspace whose coordinates are $x \in \mathbb{R}^{4}$ and $\theta_{A}^{\alpha} \in \Pi \mathbb{C}^{4}$, where $\alpha=1,2$ is the Weyl spinor index and $\Pi \mathbb{C}^{4}$ is the 4-dimensional complex space with fermionic statistics. We introduce the $\mathcal{N}=2$ chiral supermultiplet as follows:

$$
\Psi(x, \theta)=H(x)+\sqrt{2} \theta_{A} \psi^{A}(x)+\frac{1}{\sqrt{2}} \theta_{A} \sigma^{\mu \nu} \theta^{A} F_{\mu \nu}(x)+\cdots .
$$

Then the action (1) can be written as

$$
S_{\text {micro }}(X)=\frac{1}{4 \pi} \Im \mathfrak{m}\left\{\int \mathrm{d}^{4} x \mathrm{~d}^{4} \theta \frac{\tau}{2 h^{\vee}} \operatorname{Tr} \Psi^{2}(x, \theta)\right\},
$$

where

$$
\tau=\frac{4 \pi i}{g^{2}}+\frac{\Theta}{2 \pi}
$$

is the complex coupling constant.

\subsection{Low energy physics}

At low energies thanks to the Higgs potential $-\frac{1}{2}\left[H, H^{\dagger}\right]^{2}$ the Higgs field can have non-zero vacuum expectations values (vevs). Let us consider the situation when the supersymmetry remains unbroken at low energies. It follows that the ground state energy is zero. Therefore we arrive to the condition $\left[H, H^{\dagger}\right]=0$. It follows that $\langle H\rangle$ is the diagonal matrix. Mathematically speaking the vevs of Higgs belong to the Cartan subalgebra of the gauge group Lie algebra. If the rank of the algebra is $r$ then $\left\langle H_{l}\right\rangle_{a}=-2 \sqrt{2} a_{l}, l=1, \ldots, r$ and we denote by $\langle\cdot\rangle_{a}$ the vacuum expectation over the configuration satisfying such a condition.

If all $a_{l}$ are different we have the Coulomb branch of the theory. In this situation we have maximal breaking of the gauge group $G \mapsto[\mathrm{U}(1)]^{r} / W_{G}$, where $W_{G}$ is the Weyl group of the gauge group Lie algebra which is responsible for the $a_{l}$ 's permutations.

The terms $\nabla^{\mu} H^{\dagger} \nabla_{\mu} H$ and $-\frac{i}{\sqrt{2}} \psi_{A}\left[H^{\dagger}, \psi^{A}\right]+\frac{i}{\sqrt{2}} \bar{\psi}^{A}\left[H, \bar{\psi}_{A}\right]$ are responsible for the mass appearance in the gluon and gluinos field. The mass is of order of $a_{l}-a_{m}$. Therefore in the Coulomb branch the only massless states belong to the Cartan subalgebra.

Now let us consider the Wilsonian low-energy effective action defined as

$$
\mathrm{e}^{\frac{i}{\hbar} S_{\text {eff }}(\tilde{X}, \Lambda)}=\int_{|k|>\Lambda} \mathcal{D} X \mathrm{e}^{\frac{i}{\hbar} S_{\text {micro }}(X)}
$$

If $\left|a_{l}-a_{m}\right| \gg \Lambda$ when $l \neq m$ the $\tilde{X}$ consists of the fields contained in the Cartan subalgebra part of $\Psi(x, \theta)$, otherwise in $\Psi_{l}(x, \theta)$.

The unbroken supersymmetry condition implies the strong restriction on the form of the effective action. Namely one can show that [11]

$$
S_{\text {eff }}(\Psi, \Lambda)=\frac{1}{4 \pi} \Im \mathfrak{m}\left\{\frac{1}{2 \pi i} \int \mathrm{d}^{4} x \mathrm{~d}^{4} \theta \mathcal{F}(\Psi, \Lambda)\right\}+\int \mathrm{d}^{4} x \mathrm{~d}^{4} \theta \mathrm{d}^{4} \bar{\theta} \mathcal{H}(\Psi, \bar{\Psi}, \Lambda)+\cdots
$$

where $\mathcal{F}(\Psi, \Lambda)$ is an analytical function of $r+1$ variables known as prepotential, $\mathcal{H}(\Psi, \bar{\Psi}, \Lambda)$ is a real function. The leading term contains up to 2 derivatives and 4 fermions, the second contains 4 derivatives and 8 fermions and so on. Note that the leading term is the direct generalization of $(3)$. 


\subsection{Seiberg-Witten solution}

The leading term of the effective action (6) is the main object of our investigation. It is completely defined by the prepotential $\mathcal{F}(\Psi, \Lambda)$. The prepotential can be split into three parts:

$$
\mathcal{F}(\Psi, \Lambda)=\mathcal{F}^{\text {class }}(\Psi)+\mathcal{F}^{\text {pert }}(\Psi, \Lambda)+\mathcal{F}^{\text {inst }}(\Psi, \Lambda)
$$

The first term is the classical prepotential, which is defined by $(3)$ and equals $\mathcal{F}^{\text {class }}(\Psi)=$ $\pi i \tau_{0} \sum_{l=1}^{r} \Psi_{l}^{2}$. The perturbative contributions are purely of 1-loop nature, and therefore can be computed by 1-loop Feynman diagrams. The result is

$$
\begin{aligned}
\mathcal{F}^{\text {pert }}(\Psi, \Lambda) & =-\sum_{\alpha \in \Delta^{+}} \boldsymbol{k}_{\Lambda}(\langle\Psi, \alpha\rangle)+\frac{1}{2} \sum_{\varrho} \sum_{\lambda \in \boldsymbol{w}_{\varrho}} \boldsymbol{k}_{\Lambda}\left(\langle\Psi, \lambda\rangle+m_{\varrho}\right) \\
& =-\sum_{\alpha \in \Delta^{+}} \boldsymbol{k}(\langle\Psi, \alpha\rangle)+\frac{1}{2} \sum_{\varrho} \sum_{\lambda \in \boldsymbol{w}_{\varrho}} \boldsymbol{k}\left(\langle\Psi, \lambda\rangle+m_{\varrho}\right)+\frac{\beta}{2} \ln \Lambda \sum_{l=1}^{r} \Psi_{l}^{2},
\end{aligned}
$$

where

$$
\boldsymbol{k}_{\Lambda}(x)=\frac{1}{2} x^{2}\left(\ln \left|\frac{x}{\Lambda}\right|-\frac{3}{2}\right), \quad \boldsymbol{k}(x)=\boldsymbol{k}_{1}(x)=\frac{1}{2} x^{2}\left(\ln |x|-\frac{3}{2}\right),
$$

and $\beta$ in the last line is the leading (and the only) coefficient of the $\beta$-function expansion. $\Delta^{+}$ is the set of all positive roots of the gauge group Lie algebra and $\boldsymbol{w}_{\varrho}$ is the weight system of the matter multiplet representation $\varrho$ of the gauge group, $m_{\varrho}$ being its mass. Combining (7) with the classical prepotential we get the following RG-flow for the complex coupling constant:

$$
\tau(\Lambda)=\tau_{0}+\frac{\beta}{2 \pi i} \ln \Lambda .
$$

Note that $\beta$ in these formulae is always integer.

The classical theory has $\mathrm{U}(2)=\mathrm{SU}(2)_{I} \times \mathrm{U}(1)_{\mathcal{R}}$ internal symmetry. In the quantum theory due to the ABJ anomaly the last factor becomes broken down to $\mathbb{Z}_{\beta} \equiv \mathbb{Z} / \beta \mathbb{Z}$. According to this (thinking of $\Lambda$ as of a vacuum expectation value of a supplementary field with the same symmetry [11]) we get

$$
\mathcal{F}^{\text {inst }}(\Psi, \Lambda)=\sum_{k=1}^{\infty} \mathcal{F}_{k}(\Psi) \Lambda^{k \beta} .
$$

Now using (8) and (4) we conclude that

$$
\Lambda^{k \beta} \sim \mathrm{e}^{2 \pi i k \tau}=\mathrm{e}^{-\frac{8 \pi^{2} k}{g^{2}}+i k \Theta}=\mathrm{e}^{-S_{\text {micro }}\left(X_{k}\right)},
$$

where $X_{k}$ is the solution of the classical equations of motion for the $k$-instanton sector. Therefore the $\Lambda^{\beta}$-expansion of $\mathcal{F}^{\text {inst }}(\Psi, \Lambda)$ can be identified with the instanton expansion, and each term $\mathcal{F}_{k}(\Psi)$ comes from the $k$-instanton vacuum.

Seiberg and Witten in $[12,13]$ have proposed a very elegant, but rather indirect way to determine $\mathcal{F}^{\text {pert }}(\Psi, \Lambda)+\mathcal{F}^{\text {inst }}(\Psi, \Lambda)$. The following supplementary objects are needed: an algebraic curve, which is defined as a zero locus of a polynomial $\mathcal{C}(z, y, q), q=\mathrm{e}^{2 \pi i \tau}$ being the instanton counting parameter, and a meromorphic differential $\lambda(z)$ defined in such a way that its derivatives with respect to the algebraic curve moduli be holomorphic differentials. Then the prepotential is defined by the following relations

$$
a_{l}=\oint_{A_{l}} \lambda, \quad a_{D}^{l}=\frac{\partial \mathcal{F}}{\partial a_{l}}=2 \pi i \oint_{B_{l}} \lambda,
$$




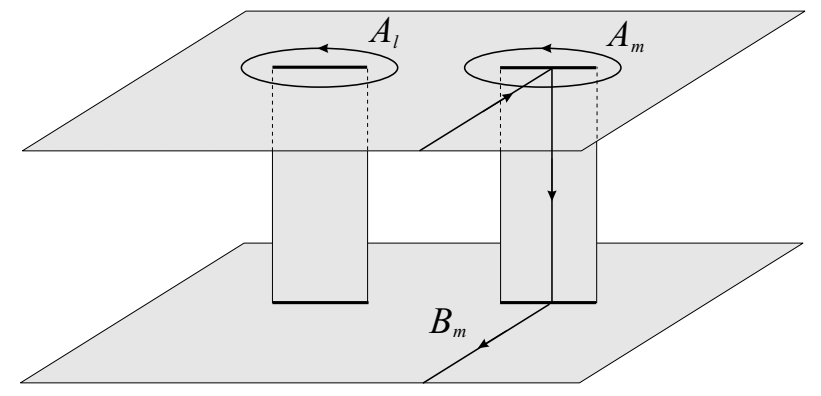

Figure 1. $A$ - and $B$-cycles.

where $A_{l}$ and $B_{l}$ are basic cycles of the algebraic curve, which can be represented as a Riemann surface with cuts (see Fig. 1). The intersection number is $A_{l} \# B_{m}=\delta_{l, m}$.

Seiberg-Witten theory allows to reduce the non-perturbative computation problem to a problem of Riemann geometry. However to use the whole power of this approach we need to know the exact form of the algebraic curve, that is, the polynomial $\mathcal{C}(z, y, q)$. Initially for the $\mathrm{SU}(2)$ case it was done using the first principles. However in more general situation it becomes too difficult. Some other methods was invented to get access to curves. Let us mention the relation with the integrable models [20], the geometrical engineering [5, 4] and the Type IIA/M-theory engineering $[19,3]$. In the last approach the Seiberg-Witten curve which was initially introduced as an auxiliary object becomes a subspace of the 11-dimensional target space.

\section{Instanton counting}

In this section we describe the Nekrasov approach to $\mathcal{N}=2$ super Yang-Mills theory. First we explain how the closed relation for the prepotential can be obtained using some peculiar properties of the microscopic action (1). When we show how the Seiberg-Witten curves can be extracted form the exact formulae.

\subsection{Localization and deformation}

In [8] a new powerful technique to compute the low-energy effective action was proposed. The idea is to perform the direct evaluation of the functional integral, which defines the partition function of the theory using the localization approach. As it was noticed for the first time in $[18,17]$ the $\mathcal{N}=2$ super Yang-Mills action is an example of the Cohomological Field Theories. Namely, one can introduce linear combinations of supercharges

$$
\overline{\mathcal{Q}}=\epsilon^{A \dot{\alpha}} \overline{\mathcal{Q}}_{A, \dot{\alpha}}, \quad \mathcal{Q}_{\mu}=\bar{\sigma}_{\mu}^{A \alpha} \mathcal{Q}_{A, \alpha}, \quad \overline{\mathcal{Q}}_{\mu \nu}=\bar{\sigma}_{\mu \nu}{ }^{A \dot{\alpha}} \overline{\mathcal{Q}}_{A, \dot{\alpha}}
$$

which, after the topological twist become scalar, vector and anti-selfdual two-form with respect to the Lorentz group. The scalar fermionic operator can be identified with the BRST operator [1] for the appropriate gauge fixing procedure for the topological action

$$
S_{\text {top }}=\frac{\Theta}{32 \pi^{2} h^{\vee}} \int \mathrm{d}^{4} x \operatorname{Tr}\left\{F_{\mu \nu} \star F^{\mu \nu}\right\},
$$

where $h^{\vee}$ is the dual Coxeter number, the trace is taken over the adjoint representation and $\star F_{\mu \nu}=\frac{1}{2} \epsilon_{\mu \nu \rho \sigma} F^{\rho \sigma}$.

Another amazing property of the $\mathcal{N}=2$ super Yang-Mills action is the existence of a Lorentz deformation which preserves one of four supercharges. Technically this deformation can be explained as follows. The $\mathcal{N}=2$ supersymmetric Yang-Mills action can be obtained as the 
compactification of $\mathcal{N}=1, d=6$ super Yang-Mills action. If we compactify $x^{4} \equiv x^{4}+2 \pi R_{4}$ and $x^{5} \equiv x^{5}+2 \pi R_{5}$ in the flat space $\mathrm{d} s_{6}^{2}=g_{\mu \nu} \mathrm{d} x^{\mu} \mathrm{d} x^{\nu}-\mathrm{d} x^{a} \mathrm{~d} x^{a}, a=4,5$, we get the undeformed theory. However if we use the following spacetime dependent metric [6]

$$
\mathrm{d} s_{6}^{2}=g_{\mu \nu}\left(\mathrm{d} x^{\mu}+V_{a}^{\mu}(x) \mathrm{d} x^{a}\right)\left(\mathrm{d} x^{\nu}+V_{b}^{\nu}(x) \mathrm{d} x^{b}\right)-\mathrm{d} x^{c} \mathrm{~d} x^{c},
$$

where $V_{a}^{\mu}(x)=\Omega_{a, \nu}^{\mu} x^{\nu}$ and $\Omega_{a, \nu}^{\mu}$ are matrices of Lorentz rotations, we get the deformed version of the theory. In this deformed version the dynamically generated scale becomes effectively superspace dependent, due to the RG-equation (8) and the following redefinition of the complex coupling constant (4)

$$
\tau(x, \theta)=\tau-\frac{1}{\sqrt{2}}\left(\left(\bar{\Omega}_{\mu \nu}\right)^{+} \theta^{\mu} \theta^{\nu}-\frac{1}{2 \sqrt{2}} \bar{\Omega}_{\mu \nu} \Omega^{\mu}{ }_{\rho} x^{\rho} x^{\nu}\right),
$$

where $\theta_{\mu}=\bar{\sigma}_{\mu}^{A \alpha} \theta_{A, \alpha}$ is twisted at the same way as (10) supercoordinates and

$$
\Omega_{\mu \nu}=\frac{1}{\sqrt{2}}\left(\Omega_{4, \mu \nu}+i \Omega_{5, \mu \nu}\right), \quad\left(\Omega_{\mu \nu}\right)^{+}=\frac{1}{2}\left(\Omega_{\mu \nu}+\star \Omega_{\mu \nu}\right) .
$$

The only survived supercharge is the supercharge which annihilates the superspace dependent complex coupling constant (12). It is

$$
\overline{\mathcal{Q}}_{\Omega}=\overline{\mathcal{Q}}+\frac{1}{2 \sqrt{2}} \Omega^{\mu}{ }_{\nu} x^{\nu} \mathcal{Q}_{\mu} .
$$

It follows that the functional integral which represents the partition function of the theory can be computed as

$$
\begin{aligned}
\langle 1\rangle_{a} & =\int \mathcal{D} X \mathrm{e}^{-S_{\text {micro }}(X)}=\int_{|k|<\Lambda} \mathcal{D} \tilde{X} \mathrm{e}^{-S_{\text {eff }}(\tilde{X})} \\
& =\exp \left\{\frac{1}{4 \pi} \Im \mathfrak{m} \frac{1}{2 \pi i} \int \mathrm{d}^{4} x \mathrm{~d}^{4} \theta \mathcal{F}(-2 \sqrt{2} a, \Lambda(x, \theta))\right\}=\exp \frac{1}{\varepsilon_{1} \varepsilon_{2}} \mathcal{F}\left(a, \Lambda, \varepsilon_{1}, \varepsilon_{2}\right),
\end{aligned}
$$

where in the last line we have localized the integral over the superspace in the origin using the Duistermaat-Heckman formula [2] (which is also based on the idea of localization), and we have used the fact that the prepotential is an homogeneous function of degree 2 , also we have renormalized the ultraviolet cut-off [8].

The deformation parameters $\varepsilon_{1}$ and $\varepsilon_{2}$ are contained in the Lorentz rotation matrix as follows

$$
\Omega_{\mu \nu}=\frac{1}{\sqrt{2}}\left(\begin{array}{cccc}
0 & 0 & 0 & \varepsilon_{1} \\
0 & 0 & \varepsilon_{2} & 0 \\
0 & -\varepsilon_{2} & 0 & 0 \\
-\varepsilon_{1} & 0 & 0 & 0
\end{array}\right) .
$$

Another way to compute the partition function is to note that the microscopic action of the deformed theory is, up to the topological term (11), $\overline{\mathcal{Q}}_{\Omega}$-exact. It allows us to localize the whole Feynman integral on the instanton moduli space $[18,8]$. The typical expression is given by a sum over the different instanton sectors:

$$
\langle 1\rangle_{a}=Z^{\text {pert }}\left(a, \Lambda, \varepsilon_{1}, \varepsilon_{2}\right)\left(1+\sum_{k=1}^{\infty} q^{k} Z_{k}\left(a, \varepsilon_{1}, \varepsilon_{2}\right)\right),
$$

where $Z_{k}\left(a, \varepsilon_{1}, \varepsilon_{2}\right)$ can be seen as the equivariant Euler characteristics of the $k$-instanton moduli space. 
The advantage of this method is that by combining (13) and (14) we get the direct access to the prepotential, which can be represented by a contour integral of a rational function of some auxiliary variables, and can be (in principle) computed by residues. For example, the equivariant Euler characteristics of the $k$-instanton sector for the pure $\mathrm{SU}(N)$ theory is given by the following expression $\left(\varepsilon_{+}=\frac{\varepsilon_{1}+\varepsilon_{2}}{2}\right)$ :

$$
\begin{aligned}
Z_{k}\left(a, \varepsilon_{1}, \varepsilon_{2}\right)= & \frac{1}{k !} \frac{\left(\varepsilon_{1}+\varepsilon_{2}\right)^{k}}{\varepsilon_{1}^{k} \varepsilon_{2}^{k}} \oint \prod_{i=1}^{k} \frac{\mathrm{d} \phi_{i}}{2 \pi i} \prod_{i \neq j} \frac{\left(\phi_{i}-\phi_{j}\right)\left(\phi_{i}-\phi_{j}-\varepsilon_{1}-\varepsilon_{2}\right)}{\left(\phi_{i}-\phi_{j}-\varepsilon_{1}\right)\left(\phi_{i}-\phi_{j}-\varepsilon_{2}\right)} \\
& \times \prod_{i=1}^{k} \prod_{l=1}^{N} \frac{1}{\left(\phi_{i}-a_{l}-\varepsilon_{+}\right)\left(\phi_{i}-a_{l}+\varepsilon_{+}\right)} .
\end{aligned}
$$

In [8] it was shown how to compute this integral while taking into account the combinatorics of residues.

\subsection{Thermodynamical limit}

Even though we have got an exact expression for the prepotential, that is, for the low-energy effective action, this expression is a series over the ultraviolet cut-off $\Lambda$. However, to study such non-perturbative effects as confinement and the monopole and dyon condensation we should be able to make an analytical continuation beyond the convergence radius. A method which can help us is the Seiberg-Witten theory. Recall that the prepotential which is defined by an algebraic curve can be defined for any $\Lambda$. Therefore should we have a curve, the continuation can be easily obtained.

So now we are faced to the "inverse problem": we know the series on $\Lambda$ around zero and we wish to reconstruct the exact Seiberg-Witten curve. This problem was solved in [9]. The idea is that in fact we are interested in the non-deformed theory. Therefore we can think of $\varepsilon_{1}$ and $\varepsilon_{2}$ as of small parameters. One can show that in the limit $\varepsilon_{1} \varepsilon_{2} \rightarrow 0$ the whole sum (14) is dominated by a single term with $k \sim \frac{1}{\varepsilon_{1} \varepsilon_{2}} \rightarrow \infty$. This effect can be illustrated by the following example: consider the series for the exponent $\mathrm{e}^{x}$. When $x \rightarrow \infty$ the whole sum is dominated by a single term with $k \sim x$. Indeed, we have

$$
\mathrm{e}^{x}=\sum_{k=0}^{\infty} \frac{x^{k}}{k !} \sim \frac{x^{x}}{x !}
$$

which is another way to claim the Stirling's formula.

Another observation is that when $k \rightarrow \infty$ the $k$-tuple integration can be replaced by the functional integration over the instanton density

$$
\rho(x)=\varepsilon_{1} \varepsilon_{2} \sum_{i=1}^{k} \delta\left(x-\phi_{i}\right) .
$$

Formulae get simple if instead of the instanton density we introduce the profile function

$$
f(x)=\sum_{l=1}^{N}\left|x-a_{l}\right|-2 \rho(x) .
$$

This function possess the following features (which can be proved quite straightforwardly)

$$
\frac{1}{2} \int_{\mathbb{R}} \mathrm{d} x f^{\prime \prime}(x)=N, \quad \frac{1}{2} \int_{\mathbb{R}} \mathrm{d} x f^{\prime \prime}(x) x=\sum_{l=1}^{N} a_{l}=0,
$$




$$
\frac{1}{2} \int_{\mathbb{R}} \mathrm{d} x f^{\prime \prime}(x) x^{2}=\sum_{l=1}^{N} a_{l}^{2}-2 \varepsilon_{1} \varepsilon_{2} k .
$$

The last equation fixes the relation between $k$ and $\varepsilon_{1} \varepsilon_{2}$.

Then the partition function can be represented in this limit as follows:

$$
\langle 1\rangle_{a} \sim \int \mathcal{D} f \exp \left\{-\frac{1}{\varepsilon_{1} \varepsilon_{2}}\left(H[f]+\frac{\pi i \tau(\Lambda)}{2} \int \mathrm{d} x f^{\prime \prime}(x) x^{2}+O\left(\varepsilon_{1}, \varepsilon_{2}\right)\right)\right\} .
$$

The Hamiltonian $H[f]$ can be reconstructed with the help of the exact formula for the prepotential. Consider, for example, the $\mathrm{SU}(N)$ model without matter hypermultiplets. The exact expression is given by (15). We have

$$
\begin{aligned}
& \prod_{i \neq j} \frac{\left(\phi_{i}-\phi_{j}\right)\left(\phi_{i}-\phi_{j}-\varepsilon_{1}-\varepsilon_{2}\right)}{\left(\phi_{i}-\phi_{j}-\varepsilon_{1}\right)\left(\phi_{i}-\phi_{j}-\varepsilon_{2}\right)} \sim \exp \left(-\varepsilon_{1} \varepsilon_{2} \sum_{i \neq j} \frac{1}{\left(\phi_{i}-\phi_{j}\right)^{2}}\right) \\
& =\exp \left(-\frac{1}{\varepsilon_{1} \varepsilon_{2}} \int \mathrm{d} x \mathrm{~d} y \frac{\rho(x) \rho(y)}{(x-y)^{2}}\right)=\exp \left(\frac{1}{\varepsilon_{1} \varepsilon_{2}} \int \mathrm{d} x \mathrm{~d} y \rho^{\prime \prime}(x) \rho^{\prime \prime}(y) \boldsymbol{k}(x-y)\right), \\
& \prod_{i=1}^{k} \prod_{l=1}^{N} \frac{1}{\left(\phi_{i}-a_{l}-\varepsilon_{+}\right)\left(\phi_{i}-a_{l}+\varepsilon_{+}\right)} \sim \exp \left(-2 \sum_{i=1}^{k} \sum_{l=1}^{N} \ln \left(\phi_{i}-a_{l}\right)\right) \\
& =\exp \left(-\frac{2}{\varepsilon_{1} \varepsilon_{2}} \sum_{l=1}^{N} \int \mathrm{d} x \rho(x) \ln \left(x-a_{l}\right)\right)=\exp \left(-\frac{2}{\varepsilon_{1} \varepsilon_{2}} \sum_{l=1}^{N} \int \mathrm{d} x \rho^{\prime \prime}(x) \boldsymbol{k}\left(x-a_{l}\right)\right) .
\end{aligned}
$$

Using (7), (8) and (16) the perturbative contribution to the partition function can be rewritten as follows

$$
\begin{aligned}
& Z^{\text {pert }}(a, m, \Lambda ; \varepsilon) q^{k}=Z^{\text {pert }}(a, m, \Lambda ; \varepsilon) \Lambda^{\beta k} \mathrm{e}^{2 \pi i k \tau_{0}} \\
& \quad=\exp \frac{1}{\varepsilon_{1} \varepsilon_{2}}\left(\mathcal{F}^{\text {class }}(a, m)+\mathcal{F}^{\text {pert }}(a, m, 1)-\frac{\pi i}{2} \tau(\Lambda) \int \mathrm{d} x f^{\prime \prime}(x) x^{2}+O\left(\varepsilon_{1}, \varepsilon_{2}\right)\right) .
\end{aligned}
$$

Having combined these three formulae we finally get

$$
\begin{aligned}
q^{k} Z^{\text {pert }} Z_{k} \sim & \int \mathcal{D} f \exp \left\{-\frac{1}{\varepsilon_{1} \varepsilon_{2}}\left(-\frac{1}{4} \int \mathrm{d} x \mathrm{~d} y f^{\prime \prime}(x) f^{\prime \prime}(y) \boldsymbol{k}(x-y)\right.\right. \\
& \left.\left.+\frac{\pi i \tau(\Lambda)}{2} \int \mathrm{d} x f^{\prime \prime}(x) x^{2}\right)\right\} .
\end{aligned}
$$

The steps can be performed in the general case. We have put corresponding contributions to the Hamiltonians $H[f]$ into the Table $1, m$ being the mass of the matter hypermultiplet.

After all manipulations we arrive to the following picture: the main contribution to the prepotential is given by the minimizer $f_{\star}(x)$ of the Hamiltonian. The supporter of this minimizer is a union of some disjoint intervals $\gamma_{l} \ni a_{l}$, and on these intervals the following equation holds:

$$
\frac{1}{\pi i} \frac{\delta H[f]}{\delta f^{\prime}(t)}=\xi_{l}+t \tau(\Lambda), \quad t \in \gamma_{l},
$$

where $\tau(\Lambda)$ is given by (8).

This equation can be recast into a difference equation for the primitive of the partition function resolvent

$$
F(z)=\frac{1}{4 \pi i} \int_{\mathbb{R}} \mathrm{d} x f^{\prime \prime}(x) \ln (z-x) .
$$


Table 1. Hamiltonians.

\begin{tabular}{|c|c|c|}
\hline Group & Multiplet & Contribution to $\boldsymbol{H}[f]$ \\
\hline \multirow{5}{*}{$\mathrm{SU}(N)$} & Adjoint, gauge & $-\frac{1}{4} \int \mathrm{d} x \mathrm{~d} y f^{\prime \prime}(x) f^{\prime \prime}(y) \boldsymbol{k}(x-y)$ \\
\hline & Fundamental & $\frac{1}{2} \int \mathrm{d} x f^{\prime \prime}(x) \boldsymbol{k}(x+m)$ \\
\hline & Symmetric & $\frac{1}{8} \int \mathrm{d} x \mathrm{~d} y f^{\prime \prime}(x) f^{\prime \prime}(y) \boldsymbol{k}(x+y+m)+\int \mathrm{d} x f^{\prime \prime}(x) \boldsymbol{k}(x+m / 2)$ \\
\hline & Antisymmetric & $\frac{1}{8} \int \mathrm{d} x \mathrm{~d} y f^{\prime \prime}(x) f^{\prime \prime}(y) \boldsymbol{k}(x+y+m)-\int \mathrm{d} x f^{\prime \prime}(x) \boldsymbol{k}(x+m / 2)$ \\
\hline & Adjoint, matter & $\frac{1}{4} \int \mathrm{d} x \mathrm{~d} y f^{\prime \prime}(x) f^{\prime \prime}(y) \boldsymbol{k}(x-y+m)$ \\
\hline \multirow{3}{*}{$\mathrm{SO}(N)$} & Adjoint, gauge & $-\frac{1}{8} \int \mathrm{d} x \mathrm{~d} y f^{\prime \prime}(x) f^{\prime \prime}(y) \boldsymbol{k}(x+y)+\int \mathrm{d} x f^{\prime \prime}(x) \boldsymbol{k}(x)$ \\
\hline & Fundamental & $\frac{1}{2} \int \mathrm{d} x f^{\prime \prime}(x) \boldsymbol{k}(x+m)$ \\
\hline & Adjoint, matter & $\frac{1}{8} \int \mathrm{d} x \mathrm{~d} y f^{\prime \prime}(x) f^{\prime \prime}(y) \boldsymbol{k}(x+y+m)-\int \mathrm{d} x f^{\prime \prime}(x) \boldsymbol{k}(x+m / 2)$ \\
\hline \multirow{4}{*}{$\operatorname{Sp}(N)$} & Adjoint, gauge & $-\frac{1}{8} \int \mathrm{d} x \mathrm{~d} y f^{\prime \prime}(x) f^{\prime \prime}(y) \boldsymbol{k}(x+y)-\int \mathrm{d} x f^{\prime \prime}(x) \boldsymbol{k}(x)$ \\
\hline & Fundamental & $\frac{1}{2} \int \mathrm{d} x f^{\prime \prime}(x) \boldsymbol{k}(x+m)$ \\
\hline & Antisymmetric & $\frac{1}{8} \int \mathrm{d} x \mathrm{~d} y f^{\prime \prime}(x) f^{\prime \prime}(y) \boldsymbol{k}(x+y+m)-\int \mathrm{d} x f^{\prime \prime}(x) \boldsymbol{k}(x+m / 2)$ \\
\hline & Adjoint, matter & $\frac{1}{8} \int \mathrm{d} x \mathrm{~d} y f^{\prime \prime}(x) f^{\prime \prime}(y) \boldsymbol{k}(x+y+m)+\int \mathrm{d} x f^{\prime \prime}(x) \boldsymbol{k}(x+m / 2)$ \\
\hline
\end{tabular}

The solution of the difference equation allows us to reconstruct the Seiberg-Witten curve and the Seiberg-Witten differential as follows. The curve $\mathcal{C}(z, y, q)$ and the differential $\lambda(z)$ are given by the dependence $y(z, q)$, where

$$
y(z, q)=\exp 2 \pi i F(z, q), \quad \lambda(z)=\frac{1}{2 \pi i} z \frac{\mathrm{d} y(z)}{y(z)}=z \mathrm{~d} F(z, q) .
$$

The expressions for the Hamiltonians are put into the Table 1.

\section{Results}

Now let us briefly discuss obtained results $[8,9,10,16,15,14]$. First of all let us mention that for all cases allowed by the asymptotic freedom (Table 2) the integral expressions similar to (15) are obtained. In some cases [9] these integrals can be computed by residues. However in the general case the combinatorics of residues is too complicated. The discussion about what is happening in the case of $\mathrm{SO}(N), \mathrm{Sp}(N)$ and antisymmetric representation of $\mathrm{SU}(N)$ can be found in [7].

However for all considered cases the difference equations for $F(z)$ are obtained. In some cases they are solved explicitly. Moreover an approximative method which allows to provide the 1-instanton correction is developed. It is shown that it is consistent with the localization results at the 1-instanton level.

Both the exact solutions and the 1-instanton approximations are checked against the known expression for the Seiberg-Witten curve. 
Table 2. Models accepted by the asymptotic freedom condition.

- $\underline{\mathrm{SU}(N)}$ :

- $N_{f}$ fundamental multiplets, $N_{f} \leq 2 N$,

- 1 antisymmetric multiplet and $N_{f}$ fundamentals, $N_{f} \leq N+2$,

- 1 antisymmetric multiplet and $N_{f}$ fundamentals, $N_{f} \leq N-2$,

- 2 antisymmetric multiplets and $N_{f}$ fundamentals, $N_{f} \leq 4$,

- 1 symmetric multiplet and 1 antisymmetric,

-1 adjoint multiplet.

- $\mathrm{SO}(N)$ :

- $N_{f}$ fundamental multiplets, $N_{f} \leq N-2$,

- 1 adjoint multiplet.

- $\operatorname{Sp}(N)$ :

- $N_{f}$ fundamental multiplets, $N_{f} \leq N+2$,

- 1 antisymmetric multiplet and $N_{f}$ fundamental, $N_{f} \leq 4$,

-1 adjoint multiplet.

Table 3. Dualities.

\begin{tabular}{||c|c|c|c|c|}
\hline \hline Group & Multiplet & Higgs & Fund. & Anti. \\
\hline \hline $\mathrm{SU}(N)$ & Symmetric, $m$ & $\vec{a}$ & $m / 2, m / 2, m / 2, m / 2$ & $m$ \\
\hline \hline \multirow{3}{*}{$\mathrm{SO}(N)$} & Adjoint, gauge & $(\diamond, \vec{a},-\vec{a})$ & $0,0,0,0$ & - \\
\cline { 2 - 5 } & Fundamental, $m$ & $(\diamond, \vec{a},-\vec{a})$ & $-m,+m$ & - \\
\cline { 2 - 5 } & Adjoint, $m$ & $(\diamond, \vec{a},-\vec{a})$ & - & $+m,-m$ \\
\hline \hline \multirow{5}{*}{$\mathrm{Sp}(N)$} & Adjoint, gauge & $(0,0, \vec{a},-\vec{a})$ & - & - \\
\cline { 2 - 5 } & Adjoint, gauge & & & - \\
\cline { 2 - 5 } & +2 fund., $m=0$ & $(\vec{a},-\vec{a})$ & - & $+m,-m$ \\
\cline { 2 - 5 } & Fundamental, $m$ & $(\vec{a},-\vec{a})$ & $+m,-m$ & $+m,-m$ \\
\cline { 2 - 5 } & Antisymmetric, $m$ & $(\vec{a},-\vec{a})$ & - & \\
\cline { 2 - 5 } & Adjoint, $m$ & $(\vec{a},-\vec{a})$ & $+m / 2,+m / 2,-m / 2,-m / 2$ & $+m$, \\
\hline
\end{tabular}

Even in the case when the exact solution of the model is not known, we can claim that if the difference equations which define the curve are the same (up to some redefinition of parameters) the same is true for their solutions. Otherwise there is a number of dualities. The list of such dualities is given by the Table 3 . As a "reference point" we have chosen the $\mathrm{SU}(N)$ models with a number of antisymmetric and fundamental matters. For $\operatorname{SO}(N)$ the notation $\diamond$ is 0 when $N$ is odd and is absent when $N$ is even.

\section{$5 \quad$ Further directions}

Now let us announce some questions which remain unsolved through our investigation:

- Find a recurrent procedure to reconstruct the whole curve starting from the functional equations.

- Find a method to solve them at once.

- The prepotential is represented as a contour integral which can in principle be done by residues. How to do it (how to handle the combinatorics of the residues)?

- Find a procedure to obtain subleading terms in $\varepsilon_{1}, \varepsilon_{2}$ development of $\langle 1\rangle_{a}$. They represent the interaction of the gauge theory with weak graviphoton field. 
[1] Baulieu L., Singer I.M., Topological Yang-Mills symmetry, Nucl. Phys. B, Proc. Suppl., 1988, V.5, $12-19$.

[2] Duistermaat J.J., Heckman G.J., On the variation in the cohomology in the symplectic form of the reduced phase space, Invent. Math., 1982, V.69, 259-269.

[3] Erlich J., Naqvi A., Randall L., The Coulomb branch of $\mathcal{N}=2$ supersymmetric product group theories from branes, Phys. Rev. D, 1998, V.58, 046002, 10 pages; hep-th/9801108.

[4] Katz S., Mayr P., Vafa C., Mirror symmetry and exact solution of $4 d n=2$ gauge theories. I, Adv. Theor. Math. Phys., 1998, V.1, 53-114; hep-th/9706110.

[5] Katz S., Klemm A., Vafa C., Geometric engineering of quantum field theories, Nucl. Phys. B, 1997, V.497, 173-195; hep-th/9609239.

[6] Losev A., Marshakov A., Nekrasov N., Small instantons, little strings and free fermions, hep-th/0302191.

[7] Mariño M., Wyllard N., A note on instanton counting for $\mathcal{N}=2$ gauge theories with classical gauge groups, JHEP, 2004, V.0405, paper 021, 23 pages; hep-th/0404125.

[8] Nekrasov N., Seiberg-Witten prepotential from instanton counting, Adv. Theor. Math. Phys., 2004, V.7, 831-864; hep-th/0206161.

[9] Nekrasov N., Okounkov A., Seiberg-Witten theory and random partitions, hep-th/0306238.

[10] Nekrasov N., Shadchin S., ABCD of instantons, Comm. Math. Phys., 2004, V.253, 359-391; hep-th/0404225.

[11] Seiberg N., Supersymmetry and nonperturbative beta functions, Phys. Lett. B, 1988, V.206, 75-87.

[12] Seiberg N., Witten E., Electric-magnetic duality, monopole condensation, and confinement in $\mathcal{N}=2$ supersymmetric Yang-Mills theory, Nucl. Phys. B, 1994, V.426, 19-52; hep-th/9407087.

[13] Seiberg N., Witten E., Monopoles, duality and chiral symmetry breaking in $\mathcal{N}=2$ supersymmetric QCD, Nucl. Phys. B, 1994, V.431, 484-550; hep-th/9408099.

[14] Shadchin S., Cubic curves from instanton counting, hep-th/0511132.

[15] Shadchin S., On certain aspects of string theory/gauge theory correspondence, PhD Thesis, Université Paris-Sud, Orsay, France, 2005; hep-th/0502180.

[16] Shadchin S., Saddle point equations in Seiberg-Witten theory, JHEP, 2004, V.0410, paper 033, 38 pages; hep-th/0408066.

[17] Witten E., Introduction to topological quantum field theories, Lectures at the Workshop on Topological Methods in Physics, ICTP, Trieste, Italy (June 1990).

[18] Witten E., Topological quantum field theory, Comm. Math. Phys., 1988, V.117, 353-386.

[19] Witten E., Solutions of four-dimensional field theories via M-theory, Nucl. Phys. B, 1997, V.500, 3-42; hep-th/9703166.

[20] Witten E., Donagi R., Supersymmetric Yang-Mills systems and integrable systems, Nucl. Phys. B, 1996, V.460, 299-334; hep-th/9510101. 